\title{
Stochastic Optimal Control of Investment and Dividend Payment Model under Debt Control with Time-Inconsistency
}

\author{
Dan Zhu $(\mathbb{D})$ and Chuancun Yin (iD) \\ School of Statistics, Qufu Normal University, Shandong Province 273165, China \\ Correspondence should be addressed to Chuancun Yin; ccyin@mail.qfnu.edu.cn
}

Received 23 April 2018; Accepted 5 June 2018; Published 9 July 2018

Academic Editor: Weihai Zhang

Copyright (C) 2018 Dan Zhu and Chuancun Yin. This is an open access article distributed under the Creative Commons Attribution License, which permits unrestricted use, distribution, and reproduction in any medium, provided the original work is properly cited.

\begin{abstract}
This paper considers the optimal debt ratio, investment, and dividend payment policies for insurers with time-inconsistency. The surplus process of an insurance company is determined by the change of asset value and the change of liabilities. The asset can be invested in financial market which contains a risky asset and a risk-free asset, and when the insurer incurs a liability, he/she earns some premium. The objective is to maximize the expected nonconstant discounted utility of dividend payment until a determinate time. This is a time-inconsistent control problem. We obtain the modified HJB equation and the closed-form expressions for the optimal debt ratio, investment, and dividend payment policies under logarithmic utility.
\end{abstract}

\section{Introduction}

In recent decades, the actuarial science has shown a trend of diversified development. We can see that the relationships between mathematics and risk theory are becoming more and more close; furthermore, a lot of optimal control research problems are becoming very interesting and increasingly important. As a result, some scholars have already studied the optimal investment, optimal reinsurance, optimal dividend, and risk control problems for decades. Specially, as an important way of profit, the optimal investment problems have been widely investigated for many years in literatures. For example, [1] considered an insurance firm that is faced with a stochastic cash flow or random risk process and studied the optimal investment policies with considering exponential utility and minimizing the probability of ruin; for other investment problems also see $[2,3]$ and so on. The optimal dividend payment has been studied for many years since it was put forward by [4]. For example, $[5,6]$ investigated the optimal dividend strategies with Brownian motion and the compound Poisson model, respectively, $[7,8]$ investigated the optimal dividend strategies under spectrally negative Lévy processes and spectrally positive Lévy processes, respectively, [9] studied optimal dividends under nonlinear insurance risk processes, [10] considered the optimal dividend strategies with capital injections and proportional transaction costs under a jump-diffusion model, and the reader can see more optimal dividend problem in a review about dividend [11]. In addition, researchers not only consider the optimal investment policies or optimal dividends individually but also combine with other optimal control problems. For example, [12-16] considered the optimal investment problems combining with reinsurance. Some researchers also investigated the investment problems with the optimal dividends policies, for example, [17-19].

Recently, the debt management problem has drawn more and more attention by many researchers. In particular, the occurrence of a global financial crisis in 2008 which was brought out by the collapse of US housing market in 2007 had drawn increasing attention to the debt ratio. It is now widely believed that excessive leveraging, and an excessive debt ratio, at key financial institutions helped convert the initial subprime turmoil in 2007 into a full blown financial crisis of 2008. In addition, the failure of AIG (American International Group) told us that the proper debt ratio in an insurance company is very important. Then, many scholars have investigated the optimal debt ratio under different risk models. For example, [20] derived the optimal debt ratio and 
dividend payment strategies with reinsurance for an insurer. The paper [21] studied the optimal investment and dividend payment strategies with debt management and reinsurance.

In this paper, we consider the optimal debt ratio, investment, and dividend payment policies for insurers with timeinconsistency. For a very rich number of scholars, they assume that the discount is constant so that the discount function is exponential. However, some scholars argue that the assumption of constant discount rate which means the unchanging of human behavior is unrealistic. Hence, many researchers have studied the so-called time-inconsistent control problems for many years. For example, [22] used Nash equilibrium points to seek the equilibrium policy in dynamic utility maximization under inconsistency; [23] discussed the consumption and portfolio rules for timeinconsistent investors; they derived a modified HJB equation and compared the different styles of investors which are called naive and sophisticated agents. Other studies about time-inconsistency can be seen in $[24,25]$ and so on. This paper assumes that the discount rate is nonconstant but that a function of time is a nonincreasing function. We assume that the surplus process of an insurance company is determined by the change of asset value and the change of liabilities. The asset can be invested in financial market which contains a risky asset and a risk-free asset, when the insurer incurs a liability, he/she earns some premium. The objective is to maximize the expected nonconstant discounted utility of dividend payment until a determinate time. Then we obtain the closed-form expressions for the optimal debt ratio, investment, and dividend payment policies under logarithmic utility. The model is a generalization of [21] in which the optimal policies with a fixed discount rate are considered. We investigate the optimal policies with a nonconstant discount rate but a nonincreasing function of time.

The remainder of this paper is as follows. Section 2 describes the model and gives the objective function and other basic foundations. In Section 3, we obtain the dynamic equation which so-called modified HJB equation. Finally, we obtain the optimal policies in Section 4.

\section{The Model}

We first give a complete probability space $(\Omega, \mathscr{F}, P)$ with filtration $\left\{\mathscr{F}_{t}\right\}_{t \in[0, T]}$ satisfying the usual condition generated by a standard Brownian motion $\{W(t), t \geq 0\}$. Let $X(t)$ denote the surplus of a large insurer. The asset value and liabilities are represented by $K(t)$ and $L(t)$, respectively. The change of surplus process, which is the difference between the change of asset value $K(t)$ and the change of liabilities $L(t)$, can be written as

$$
\begin{aligned}
\mathrm{d} X(t) & =\mathrm{d} K(t)-\mathrm{d} L(t), \\
X(0) & =x .
\end{aligned}
$$

Obviously, when the insurer incurs a liability, he earns some premium. Hence, in this paper, we assume the premium rate is $\theta$ which means that the insurer can collect $\theta$ dollar when he/she provides a dollar insurance protection. Furthermore, the asset value increasing from the insurance sales during $[t, t+\mathrm{d} t]$ is denoted by $\theta L(t) \mathrm{d} t$. Naturally, the insurer wants to know that how much $L(t)$ he can provided. In other words, he wants to know how much of the debt ratio is suitable to the company. Let $\pi_{t}=L(t) / X(t)$ denote the debt ratio of the insurance company and $\pi_{t}$ also serves as a control variable in this paper. Then, the leverage is described as the ratio between asset values and surplus can be represented as $1+\pi_{t}=K(t) / X(t)$. With some assumption, we will give the optimal debt ratio in the following.

For the sake of simplicity, we assume there are only a riskfree asset $P_{0}(t)$ and a risky asset $P_{1}(t)$ in the financial market with prices satisfying

$$
\begin{gathered}
\frac{\mathrm{d} P_{0}(t)}{P_{0}(t)}=r \mathrm{~d} t \\
\frac{\mathrm{d} P_{1}(t)}{\mathrm{P}_{1}(t)}=\mu \mathrm{d} t+\sigma \mathrm{d} W(t),
\end{gathered}
$$

where $r, \mu$, and $\sigma$ are some positive real numbers satisfying $r<\mu$. Assume that the asset is invested in financial market, and the proportional of the asset value is invested in the risky asset at time $t$ is denoted as $f_{t}$, the rest of the asset is invested in risk-free asset. Intuitively, without considering claims and dividend payment, the surplus process can be denoted by

$$
\begin{aligned}
\mathrm{d} X_{1}(t)= & {[\theta L(t)+K(t)] f_{t} \frac{\mathrm{d} P_{1}(t)}{P_{1}(t)} } \\
& +[\theta L(t)+K(t)]\left(1-f_{t}\right) \frac{\mathrm{d} P_{0}(t)}{P_{0}(t)} .
\end{aligned}
$$

Substituting (2) and (3) into (4), we have

$$
\begin{aligned}
& \mathrm{d} X_{1}(t) \\
& =[\theta L(t)+K(t)]\left\{\left[r+(\mu-r) f_{t}\right] \mathrm{d} t+\sigma f_{t} \mathrm{~d} W(t)\right\}, \\
& X_{1}(0)=x \text {. }
\end{aligned}
$$

Furthermore, we consider the future claims. We denoted by $S(t)$ the accumulated claims up to time $t$. We have that

$$
\begin{aligned}
& S(t)=\int_{0}^{t} c(s) L(s) \mathrm{d} s, \\
& S(0)=0
\end{aligned}
$$

where $c(s)$ is claim rate. This means the claims are proportional to the amount of insurance liabilities. Let $D(t)$ be a nonnegative and nondecreasing process representing the sum of the dividends distributed over the time interval $[0, t]$. In this paper, our purpose is to investigate the optimal dividend strategy where the dividend payment is proportional to amount of the surplus. Hence, the accumulate dividends can be represented by

$$
\begin{aligned}
D(t) & =\int_{0}^{t} z_{s} X(s) \mathrm{d} s, \\
D(0-) & =0,
\end{aligned}
$$


where $z_{t}$ is a dividend payment rate and is an $\mathscr{F}_{t}$ adapted process. Combining with (5) and considering claims and dividends, the surplus of insurance company is given by

$$
\begin{aligned}
& d X(t)=\mathrm{d} X_{1}(t)-\mathrm{d} S(t)-\mathrm{d} D(t)=[\theta L(t)+K(t)] \\
& \cdot\left\{\left[r+(\mu-r) f_{t}\right] \mathrm{d} t+\sigma f_{t} \mathrm{~d} W(t)\right\}-c(t) L(t) \mathrm{d} t \\
& -z_{t} X(t) \mathrm{d} t=\left\{[\theta L(t)+K(t)]\left[r+(\mu-r) f_{t}\right]\right. \\
& \left.\quad-c(t) L(t)-z_{t} X(t)\right\} \mathrm{d} t+\sigma f_{t}[\theta L(t) \\
& \quad+K(t)] \mathrm{d} W(t), \quad \text { with } X(0-)=x .
\end{aligned}
$$

Naturally, we have

$$
\begin{aligned}
& \frac{\mathrm{d} X(t)}{X(t)} \\
& \quad=\left\{\left[(1+\theta) \pi_{t}+1\right]\left[r+(\mu-r) f_{t}\right]-c(t) \pi_{t}-z_{t}\right\} \mathrm{d} t \\
& \quad+\left[(1+\theta) \pi_{t}+1\right] \sigma f_{t} \mathrm{~d} W(t) .
\end{aligned}
$$

Recall that $\pi_{t}$ denotes the debt ratio, the insurer wants to determine the optimal liabilities ratio. Thus, in our paper, $\pi_{t}$ serve as a control variable. Let $\Pi=[0, M], 0<M<\infty$. Then, we give the following definition.

Definition 1. We say that $\mathbb{V}=\left\{\pi_{t}, f_{t}, z_{t}\right\}_{t \in[0, T]}$ is an admissible set, if

(i) the process $\left\{\pi_{t}, 0 \leq t \leq T\right\}$ is a predictable and satisfies that $\pi_{t} \in \Pi$;

(ii) the process $\left\{f_{t}, 0 \leq t \leq T\right\}$ is a predictable and satisfies that

$$
E \int_{0}^{T} f_{s}^{2} \mathrm{~d} s<\infty, \quad T<\infty
$$

(iii) the process $\left\{z_{t}, 0 \leq t \leq T\right\}$ is a predictable and satisfy that $0 \leq z_{t}<\infty$.

We also say a policy $\left(\pi_{t}, f_{t}, z_{t}\right)$ is admissible, if $\left(\pi_{t}, f_{t}\right.$, $\left.z_{t}\right) \in \mathbb{V}$.

In traditional optimal strategies research, the scholars also assume that the insurer preferences at time $t$ take the form

$$
\begin{aligned}
& J_{1}(t, x)=E_{t, x}\left\{\int_{t}^{T}\left[e^{-\rho(s-t)} U\left(z_{s} X(s)\right)\right] \mathrm{d} s\right. \\
& \left.+e^{-\rho(T-t)} U(X(T))\right\},
\end{aligned}
$$

where $E_{t, x}$ denotes condition expectation $E[\cdot \mid X(t)=x]$, $U(\cdot)$ is a utility function satisfying that $U^{\prime}>0, U^{\prime \prime}<0, \rho$ is a discount rate. The insurer wants to choose the optimal policies to maximize $J_{1}(t, x)$, i.e.,

$$
V_{1}(t, x)=\max _{\left\{\pi_{t}, f_{t}, z_{t}\right\} \in \mathbb{V}} J_{1}(t, x),
$$

For the purpose of obtaining optimal policies, we ought to solve a stochastic control problem, and since the discount rate is constant, the solution is time consistent. However, some scholars argue that the assumption of constant discount rate is unrealistic. Hence, in this paper, we assume that the discount rate is nonconstant, but a function of time $v(s)$ that is a nonincreasing function for $s \in[0, T]$. So the discount factor at time $s$ used to evaluate a payoff at time $s+t, t \geq 0$, is $\varsigma(t)=\exp \left[-\int_{0}^{t} \nu(s) \mathrm{d} s\right]$. Consequently, we assume that the insurer's preference at time $t$ takes the form of

$$
\begin{aligned}
& J(t, x)=E_{t, x}\left\{\int_{t}^{T}\left[\varsigma(s-t) U\left(z_{s} X(s)\right)\right] \mathrm{d} s\right. \\
& +\varsigma(T-t) U(X(T))\},
\end{aligned}
$$

which means the insurer's preference is not immutable. The optimal debt ratio, investment, and dividend payment policies consist of solving the following stochastic control problem:

$$
V(t, x)=\max _{\left\{\pi_{t}, f_{t}, z_{t}\right\} \in \mathbb{V}} J(t, x), \quad \text { with } V(T, x)=U(x) .
$$

\section{Dynamic Equation}

For any $\left\{\pi_{t}, f_{t}, z_{t}\right\} \in \mathbb{V}$ and any real function $g(t, x) \in \mathscr{C}^{1,2}([0$, $\infty) \times[0, \infty)$ ), we define a generator $\mathscr{A}$ about (9) as

$$
\begin{aligned}
& \mathscr{A} g(t, x)=\frac{1}{2}\left[(1+\theta) \pi_{t}+1\right]^{2} \sigma^{2} f_{t}^{2} x^{2} \frac{\partial^{2}}{\partial x^{2}} g(t, x) \\
& +\left\{\left[(1+\theta) \pi_{t}+1\right]\left[r+(\mu-r) f_{t}\right]-c(t) \pi_{t}-z_{t}\right\} \\
& \cdot x \frac{\partial}{\partial x} g(t, x) .
\end{aligned}
$$

For problem (12), in the conventional methods, by dynamic programming principle, the value function satisfies HJB (Hamilton-Jacobi-Bellman) equation (see [21])

$$
\begin{array}{r}
\max _{\left\{\pi_{t}, f_{t}, z_{t}\right\} \in \mathbb{V}}\left\{\frac{\partial}{\partial t} V(t, x)+\mathscr{A} V(t, x)+e^{-\rho t} U(z x)\right\}=0, \\
\text { with terminal condition } V(T, x)=U(x) .
\end{array}
$$

For time-inconsistent problem (14), the standard HJB equation cannot be used to obtain the solution. In what follows, we use a modified HJB equation which was used in MarínSolano and Navas [23] to deal with (14).

We begin with a special discretized version of (14). We divide the $[0, T]$ into $N$ periods of constant length $\varepsilon$; in this way we identify $d s=\varepsilon$; therefore the objective of insurer in time $t=j \varepsilon(j=0,1,2, \ldots, N)$ will be

$$
\begin{aligned}
& V(j \varepsilon, X(j \varepsilon)) \\
& \quad=\max _{\left\{\pi_{k \varepsilon}, f_{k \varepsilon}, z_{k \varepsilon}\right\}} E\left[\Sigma_{i=0}^{N-j-1} \varsigma(i \varepsilon) U\left(z_{(i+j) \varepsilon} X((i+j) \varepsilon)\right) \varepsilon\right. \\
& \quad+\varsigma((N-j) \varepsilon) U(X(T))],
\end{aligned}
$$

with $V_{1}(T, x)=U(x)$. 
From (9), if the surplus is served as discrete, the surplus will have the following form:

$$
\begin{aligned}
& X(k \varepsilon+\varepsilon)=X(k \varepsilon) \\
& \quad \cdot\left\{\left\{\left[(1+\theta) \pi_{k \varepsilon}+1\right]\left[r+(\mu-r) f_{k \varepsilon}\right]-c(t) \pi_{k \varepsilon}\right.\right. \\
& \left.-z_{k \varepsilon}\right\} \varepsilon+\left[(1+\theta) \pi_{k \varepsilon}+1\right] \sigma f_{k \varepsilon}[W((k+1) \varepsilon) \\
& \quad-W(k \varepsilon)]\}
\end{aligned}
$$

with $X(k \varepsilon)$ is given and where $k=j, \ldots, N-1$. For (17), in the final period, i.e., $j=N$, we have $\mathrm{V}(\mathrm{T}, \mathrm{X}(\mathrm{T}))=\mathrm{U}(\mathrm{X}(\mathrm{T}))$. For $j=N-1$, we have

$$
\begin{aligned}
V & ((N-1) \varepsilon, X((N-1) \varepsilon)) \\
& =\max _{\left\{\pi_{(N-1) \varepsilon}, f_{(N-1) \varepsilon}, z_{(N-1) \varepsilon}\right\}} E\left[U\left(z_{(N-1) \varepsilon} X((N-1) \varepsilon)\right) \varepsilon\right. \\
& +\varsigma(\varepsilon) V(T, X(T))] \\
& =E\left[U\left(z_{(N-1) \varepsilon}^{*} X((N-1) \varepsilon)\right) \varepsilon\right. \\
& +\varsigma(\varepsilon) V(T, X(T))]_{\left\{\pi_{(N-1) \varepsilon}^{*}, f_{(N-1) \varepsilon}^{*}, z_{(N-1) \varepsilon}^{*}\right\}},
\end{aligned}
$$

with

$$
\begin{aligned}
& X(N \varepsilon)=X((N-1) \varepsilon) \\
& \cdot\left\{\left\{\left[(1+\theta) \pi_{(N-1) \varepsilon}+1\right]\left[r+(\mu-r) f_{(N-1) \varepsilon}\right]\right.\right. \\
& \left.-c(t) \pi_{(N-1) \varepsilon}-z_{(N-1) \varepsilon}\right\} \varepsilon+\left[(1+\theta) \pi_{(N-1) \varepsilon}+1\right] \\
& \left.\cdot \sigma f_{(N-1) \varepsilon}[W(N \varepsilon)-W((N-1) \varepsilon)]\right\},
\end{aligned}
$$

where $\left\{\pi_{(N-1) \varepsilon}^{*}, f_{(N-1) \varepsilon}^{*}, z_{(N-1) \varepsilon}^{*}\right\}$ is the maximizer of the right hand term of the (19). We denote

$$
\begin{aligned}
G & ((N-1) \varepsilon, X((N-1) \varepsilon)) \\
\quad & =\left.U\left(z_{(N-1) \varepsilon}^{*} X((N-1) \varepsilon)\right) \varepsilon\right|_{\left\{\pi_{(N-1) \varepsilon}^{*}, f_{(N-1) \varepsilon}^{*}\right\}} .
\end{aligned}
$$

Therefore, the optimal value of (17) can be represented as

$$
\begin{gathered}
V(j \varepsilon, X(j \varepsilon)) \\
=\max _{\left\{\pi_{j \varepsilon}, f_{j \varepsilon}, z_{j \varepsilon}\right\}} E\left[U\left(z_{j \varepsilon} X(j \varepsilon)\right) \varepsilon+H(j, \varepsilon)\right], \\
V((j+1) \varepsilon, X((j+1) \varepsilon)) \\
=E[G((j+1) \varepsilon, X((j+1) \varepsilon)) \varepsilon+H(j+1, \varepsilon)],
\end{gathered}
$$

where

$$
\begin{aligned}
H(j, \varepsilon)= & \sum_{i=1}^{N-j-1} \varsigma(i \varepsilon) G((i+j) \varepsilon, X((i+j) \varepsilon)) \varepsilon \\
& +\varsigma((N-j) \varepsilon) V(T, X(T)) .
\end{aligned}
$$

Solving $V(T, X(T))$ in (22) and (23), then using some mathematical techniques to simplify it, we have the lemma as follows.
Lemma 2. For initial wealth $x$, the equilibrium value $V(j \varepsilon$, $X(j \varepsilon))$ of (17) and (18) can be obtained as

$$
\begin{aligned}
& \varsigma((N-j-1) \varepsilon) V(j \varepsilon, X(j \varepsilon)) \\
& \quad=\max _{\left\{\pi_{j \varepsilon}, f_{j \varepsilon}, z_{j \varepsilon}\right\}} E\left\{\varsigma((N-j-1) \varepsilon) U\left(z_{j \varepsilon} X(j \varepsilon)\right) \varepsilon\right. \\
& \quad+\Sigma_{k=1}^{N-j-1}[\varsigma((N-j-1) \varepsilon) \varsigma(k \varepsilon) \\
& \quad-\varsigma((N-j) \varepsilon) \varsigma((k-1) \varepsilon)] \\
& \quad \cdot G((k+j) \varepsilon, X((k+j) \varepsilon)) \varepsilon+\varsigma((N-j) \varepsilon) \\
& \quad \cdot V((j+1) \varepsilon, X((j+1) \varepsilon))\}
\end{aligned}
$$

with $V(T, X(T))=U(X(T))$ and

$$
\begin{aligned}
& X((j+1) \varepsilon)=X(j \varepsilon) \\
& \quad \cdot\left\{\left[(1+\theta) \pi_{j \varepsilon}+1\right]\left[r+(\mu-r) f_{j \varepsilon}\right]-c(t) \pi_{j \varepsilon}\right. \\
& \left.\quad-z_{j \varepsilon}\right\} \varepsilon+X(j \varepsilon) \\
& \quad \cdot\left\{\left[(1+\theta) \pi_{j \varepsilon}+1\right] \sigma f_{j \varepsilon}[W((j+1) \varepsilon)-W(j \varepsilon)]\right\},
\end{aligned}
$$

for $j=1,2, \ldots, N-1$. Equation (25) is the equilibrium dynamic programming equation in discrete time, and we can obtain the solutions by proceeding backward in time from period $N-1$ to period 0 .

For the continuous time problem of (14), we also derive an equilibrium dynamic programming equation called modified HJB equation. Inspired by the discrete case, the equilibrium value of (14) is defined as the limit when $\varepsilon \longrightarrow 0$ of discrete time equilibrium dynamic programming equation (25). Since $t=j \varepsilon, T=N \varepsilon$, with initial wealth $X(t)=x$, then $V(t, x)=$ $V(j \varepsilon, X(j \varepsilon))$, and by the Itô formula, we have

$$
\begin{aligned}
& V(t+\varepsilon, X(t+\varepsilon))=V(t, X(t)) \\
& +\left[\frac{\partial}{\partial t} V(t, X(t))+\mathscr{A} V(t, X(t))\right] \varepsilon \\
& +\left[(1+\theta) \pi_{t}+1\right] \sigma f_{t} \frac{\partial}{\partial x} V(t, X(t)) \\
& \quad \cdot[W(t+\varepsilon)-W(t)]+o(\varepsilon) .
\end{aligned}
$$

Since $\varsigma(k \varepsilon)=\exp \left[-\int_{0}^{k \varepsilon} \nu(s) \mathrm{d} s\right]$, by Taylor's theorem, then

$$
\begin{aligned}
& \varsigma((N-j) \varepsilon)=\exp \left[-\int_{0}^{(N-j) \varepsilon} \nu(s) \mathrm{d} s\right] \\
& =\varsigma((N-j-1) \varepsilon)[1-\nu((N-j-1) \varepsilon) \varepsilon] \\
& \quad+o(\varepsilon), \\
& \varsigma((k-1) \varepsilon)=\exp \left[-\int_{0}^{(k-1) \varepsilon} v(s) \mathrm{d} s\right] \\
& =\varsigma(k \varepsilon)[1+\nu(k \varepsilon) \varepsilon]+o(\varepsilon) .
\end{aligned}
$$


Substituting (27), (28) and (29) into (25), we have

$$
\begin{aligned}
0= & \max _{\left\{\pi_{t}, f_{t}, z_{t}\right\}} E\left\{U\left(z_{t} X(t)\right)+\frac{\partial}{\partial t} V(t, X(t))\right. \\
& +\mathscr{A} V(t, X(t))-v(T-t) V(t, X(t)) \\
& -H(t, X(t))\} \varepsilon+\left[(1+\theta) \pi_{t}+1\right] \sigma f_{t} \frac{\partial}{\partial x} \\
& \cdot V(t, X(t))[W(t+\varepsilon)-W(t)]+o(\varepsilon),
\end{aligned}
$$

where

$$
\begin{aligned}
& H(t, X(t))=E\left[\Sigma_{k=1}^{N-j-1} \varsigma(k \xi)\right. \\
& \quad \cdot(\nu(k \varepsilon)-v((N-j-1) \varepsilon)) G(t+k \varepsilon, X(t+k \varepsilon)) \\
& \quad \cdot \varepsilon]
\end{aligned}
$$

Dividing (30) by $\varepsilon$, and letting $\varepsilon \longrightarrow 0$ in (30) and (31), we derive the modified HJB equation as

$$
\begin{gathered}
v(T-t) V(t, x)-\frac{\partial}{\partial t} V(t, x)+H(t, x) \\
=\max _{\left\{\pi_{t}, f_{t}, z_{t}\right\}}\left\{U\left(z_{t} x\right)+\mathscr{A} V(t, x)\right\},
\end{gathered}
$$

where

$$
H(t, x)=E\left[\int_{0}^{T-t} \varsigma(s)[\nu(s)-v(T-t)]\right.
$$

$$
\begin{aligned}
& \cdot G(t+s, X(t+s))] d s=E\left[\int_{t}^{T} \varsigma(s-t)\right. \\
& \cdot[v(s-t)-v(T-t)] G(s, X(s))] d s .
\end{aligned}
$$

Therefore, we have the following theorem.

Theorem 3. If $v(t, x) \in \mathscr{C}^{1,2}$ is the solution to modified HJB equation (32) with $H$ satisfying (33) and with the boundary condition $v(T, X(T))=U(X(T))$, and there exists an admissible $\left\{\pi_{t}, f_{t}, z_{t}\right\}$ that solves the right side of (32), then $v(t, x)=V(t, x)$.

Proof. From Dynkin's formula, we have

$$
\begin{aligned}
& E_{t, x} {[v(T, X(T))-v(t, X(t))] } \\
&=E_{t, x}\left\{\int_{t}^{T}\left[\frac{\partial}{\partial t} v(s, X(s))+\mathscr{A} v(s, X(s))\right] d s\right\},
\end{aligned}
$$

and combining with $v(T, X(T))=U(X(T))$, we obtain

$$
\begin{aligned}
& v(t, x) \\
& \quad=E_{t, x}\left\{\int_{t}^{T}\left[-\frac{\partial}{\partial s} v(s, X(s))-\mathscr{A} v(s, X(s))\right] d s\right. \\
& \quad+U(X(T))\} .
\end{aligned}
$$

Assuming that $\left\{\pi_{t}^{*}, f_{t}^{*}, z_{t}^{*}\right\}$ satisfies the condition in Definition 1 and solving the right side of (32), then

$$
v(t, x)=\left.E_{t, x}\left\{\int_{t}^{T}\left[U\left(z_{s} X(s)\right)-v(T-s) v(s, X(s))-H(s, X(s))\right] d s+U(X(T))\right\}\right|_{\left\{\pi_{t}^{*}, f_{t}^{*}, z_{t}^{*}\right\}},
$$

where

$$
\left.E_{t, x}\left\{\int_{t}^{T} H(s, X(s)) d s\right\}\right|_{\left\{\pi_{t}^{*}, f_{t}^{*}, z_{t}^{*}\right\}}=\left.E_{t, x}\left\{\int_{t}^{T} \int_{s}^{T} \varsigma(\kappa-s)[\nu(\kappa-s)-\nu(T-s)] G(\kappa, X(\kappa)) d \kappa d s\right\}\right|_{\left\{\pi_{t}^{*}, f_{t}^{*}, z_{t}^{*}\right\}} .
$$

We know that

$$
\begin{aligned}
& E_{t, x}\left\{\int_{t}^{T} \nu(T-s)\right. \\
& \left.\cdot \int_{s}^{T} \varsigma(\kappa-s) G(\kappa, X(\kappa)) d \kappa d s\right\}\left.\right|_{\left\{\pi_{t}^{*}, f_{t}^{*}, z_{t}^{*}\right\}} \\
& =E_{t, x}\left\{\int_{t}^{T} \nu(T-s)\right.
\end{aligned}
$$

$$
\begin{aligned}
& \cdot[v(s, X(s))-\varsigma(T-s) U(X(T)) d s]\}\left.\right|_{\left\{\pi_{t}^{*}, f_{t}^{*}, z_{t}^{*}\right\}} \\
& =E_{t, x}\left\{\int_{t}^{T} v(T-s) v(s, X(s)) d s-U(X(T))\right. \\
& +\varsigma(T-t) U(X(T))\}\left.\right|_{\left\{\pi_{t}^{*}, f_{t}^{*}, z_{t}^{*}\right\}},
\end{aligned}
$$


and

$$
\begin{gathered}
\left.E_{t, x}\left\{\int_{t}^{T} \int_{s}^{T} \varsigma(\kappa-s) \nu(\kappa-s) G(\kappa, X(\kappa)) d \kappa d s\right\}\right|_{\left\{\pi_{t}^{*}, f_{t}^{*}, z_{t}^{*}\right\}} \\
=\left.E_{t, x}\left\{\int_{t}^{T} G(\kappa, X(\kappa)) \int_{t}^{\kappa} \varsigma(\kappa-s) \nu(\kappa-s) d s d \kappa\right\}\right|_{\left\{\pi_{t}^{*}, f_{t}^{*}, z_{t}^{*}\right\}}
\end{gathered}
$$$$
=\left.E_{t, x}\left\{\int_{t}^{T} G(\kappa, X(\kappa))[1-\varsigma(\kappa-t)] d \kappa\right\}\right|_{\left\{\pi_{t}^{*}, f_{t}^{*}, z_{t}^{*}\right\}} .
$$

Substituting (37), (38), and (39) into (36), we obtain

$$
\begin{aligned}
v(t, x) & =\left.E_{t, x}\left\{\int_{t}^{T}\left[U\left(z_{s} X(s)\right)-G(s, X(s))(1-\varsigma(s-t))\right] d s+\varsigma(T-t) U(X(T))\right\}\right|_{\left\{\pi_{t}^{*}, f_{t}^{*}, z_{t}^{*}\right\}} \\
& =\left.E_{t, x}\left\{\int_{t}^{T} \varsigma(s-t) U\left(z_{s} X(s)\right) d s+\varsigma(T-t) U(X(T))\right\}\right|_{\left\{\pi_{t}^{*}, f_{t}^{*}, z_{t}^{*}\right\}}=V(t, x) .
\end{aligned}
$$

This ends the proof of Theorem 3.

\section{Optimal Policies}

Suppose that the insurer has a log-utility

$$
U(x)=\ln (x),
$$

Then the modified HJB equation (32) can be rewritten as

$$
\begin{aligned}
v(T-t) V(t, x)-\frac{\partial}{\partial t} V(t, x)+H(t, x) \\
=\max _{\left\{\pi_{t}, f_{t}, z_{t}\right\}}\left\{\ln \left(z_{t} x\right)+\frac{1}{2}\left[(1+\theta) \pi_{t}+1\right]^{2}\right. \\
\cdot \sigma^{2} f_{t}^{2} x^{2} \frac{\partial^{2}}{\partial x^{2}} V(t, x) \\
\quad+\left\{\left[(1+\theta) \pi_{t}+1\right]\left[r+(\mu-r) f_{t}\right]-c(t) \pi_{t}-z_{t}\right\} \\
\left.\cdot x \frac{\partial}{\partial x} V(t, x)\right\},
\end{aligned}
$$

with boundary conditions $V(T, x)=\ln (x)$.

With the log-utility function, we speculate that the value function has the following form:

$$
V(t, x)=A(t) \ln (x)+B(t) .
$$

Then

$$
\begin{aligned}
\frac{\partial}{\partial t} V(t, x) & =A^{\prime}(t) \ln (x)+B^{\prime}(t), \\
\frac{\partial}{\partial x} V(t, x) & =\frac{A(t)}{x}, \\
\frac{\partial^{2}}{\partial t^{2}} V(t, x) & =-\frac{A(t)}{x^{2}} .
\end{aligned}
$$

Substituting (43) and (44) into (42), we have

$$
\begin{aligned}
& v(T-t)[A(t) \ln (x)+B(t)]-\left[A^{\prime}(t) \ln (x)+B^{\prime}(t)\right] \\
& +H(t, x)=\max _{\left\{\pi_{t}, f_{t}, z_{t}\right\}}\left\{\ln \left(z_{t} x\right)-\frac{1}{2}\left[(1+\theta) \pi_{t}+1\right]^{2}\right. \\
& \cdot \sigma^{2} f_{t}^{2} A(t) \\
& +\left\{\left[(1+\theta) \pi_{t}+1\right]\left[r+(\mu-r) f_{t}\right]-c(t) \pi_{t}-z_{t}\right\} \\
& \cdot A(t)\} .
\end{aligned}
$$

For simplicity, we denote $\Pi_{t}:=(1+\theta) \pi_{t}+1$, intuitively $\pi_{t}=$ $\left(\Pi_{t}-1\right) /(1+\theta)$; hence,

$$
\begin{gathered}
v(T-t)[A(t) \ln (x)+B(t)]-\left[A^{\prime}(t) \ln (x)+B^{\prime}(t)\right] \\
+H(t, x)=\max _{\left\{\pi_{t}, f_{t}, z_{t}\right\}}\left\{\ln \left(z_{t} x\right)-\frac{1}{2} \Pi_{t}^{2} \sigma^{2} f_{t}^{2} A(t)\right. \\
+\left\{\Pi_{t}\left[r+(\mu-r) f_{t}\right]-\frac{c(t)\left(\Pi_{t}-1\right)}{1+\theta}-z_{t}\right\} \\
\cdot A(t)\}=\max _{\left\{\pi_{t}, f_{t}\right\}}\left\{-\frac{1}{2} \Pi_{t}^{2} \sigma^{2}\left(f_{t}-\frac{\mu-r}{\Pi_{t} \sigma^{2}}\right)^{2}\right. \\
\left.+\Pi_{t}\left(r-\frac{c(t)}{1+\theta}\right)\right\} A(t)+\max _{\left\{z_{t}\right\}}\left\{\ln \left(z_{t} x\right)\right. \\
\left.-z_{t} A(t)\right\}+\left[\frac{\mu-r}{2 \sigma^{2}}+\frac{c(t)}{1+\theta}\right] A(t) .
\end{gathered}
$$

From (46), we can obtain that

$$
\begin{aligned}
& f_{t}^{*}=\frac{\mu-r}{\Pi_{t} \sigma^{2}}, \\
& z_{t}^{*}=\frac{1}{A(t)} .
\end{aligned}
$$

Moreover, if $r>c(t) /(1+\theta)$, then

$$
\begin{aligned}
\pi_{t}^{*} & =M, \\
\Pi_{t}^{*} & =(1+\theta) M+1 ;
\end{aligned}
$$


if $r<c(t) /(1+\theta)$, then

$$
\begin{aligned}
& \pi_{t}^{*}=0, \\
& \Pi_{t}^{*}=1 ;
\end{aligned}
$$

if $r=c(t) /(1+\theta)$, then $\pi_{t}^{*}$ can take any value in $[0, M]$. By (48),

$$
\begin{gathered}
H(t, x)=E\left[\int_{t}^{T} \varsigma(s-t)[\nu(s-t)-v(T-t)]\right. \\
\left.\cdot \ln \left(z_{s}^{*} X(s)\right)\right] d s=E\left[\int_{t}^{T} \varsigma(s-t)\right. \\
\left.\cdot[\nu(s-t)-\nu(T-t)] \ln \left(\frac{X(s)}{A(s)}\right)\right] d s .
\end{gathered}
$$

From (9), we know that

$$
X(s)=X(t) \exp \left\{\triangle_{t}(s)\right\}
$$

with

$$
\begin{aligned}
& \triangle_{t}(s)=\int_{t}^{s}\left\{\left[(1+\theta) \pi_{\iota}^{*}+1\right]\left[r+(\mu-r) f_{\iota}^{*}\right]\right. \\
& \left.-c(t) \pi_{\iota}-z_{\iota}^{*}-\frac{1}{2}\left[(1+\theta) \pi_{\iota}^{*}+1\right]^{2} \sigma^{2} f_{\iota}^{* 2}\right\} \mathrm{d} \iota \\
& +\int_{t}^{s}\left[(1+\theta) \pi_{\iota}^{*}+1\right] \sigma f_{\iota}^{*} \mathrm{~d} W(\iota) .
\end{aligned}
$$

Combining (52) and (51), we have

$$
\begin{aligned}
H(t, x)= & E \int_{t}^{T} \varsigma(s-t)[\nu(s-t)-v(T-t)] \\
& \cdot\left(\ln X(t)+\triangle_{t}(s)-\ln A(s)\right) d s .
\end{aligned}
$$

Inserting (54) into (46), we obtain that

$$
\begin{aligned}
& \left\{\nu(T-t) A(t)-A^{\prime}(t)-1\right. \\
& \left.\quad+\int_{t}^{T} \varsigma(s-t)[\nu(s-t)-v(T-t)] d s\right\} \ln x \\
& \quad=-v(T-t) B(t)-B^{\prime}(t) \\
& \quad-\int_{t}^{T} \varsigma(s-t)[\nu(s-t)-v(T-t)]\left(\triangle_{t}(s)\right. \\
& \quad-\ln A(s)) d s+\Pi_{t}^{*}\left(r-\frac{c(t)}{1+\theta}\right) A(t)-\ln A(t) \\
& \quad-1+\left[\frac{\mu-r}{2 \sigma^{2}}+\frac{c(t)}{1+\theta}\right] A(t) .
\end{aligned}
$$

Equation (55) should be satisfied for any $x$; therefore,

$$
\begin{aligned}
& v(T-t) A(t)-A^{\prime}(t)-1 \\
& \quad+\int_{t}^{T} \varsigma(s-t)[v(s-t)-v(T-t)] d s=0,
\end{aligned}
$$

and using the boundary condition $A(T)=1$, we get

$$
A(t)=\varsigma(T-t)+\int_{t}^{T} \varsigma(s-t) d s .
$$

So far, we have proved the following theorem.

Theorem 4. For problem (14), if the insurer has a log-utility function (41), we can obtain the optimal policies as follows:

$$
\begin{aligned}
& f_{t}^{*}=\frac{\mu-r}{\Pi_{t}^{*} \sigma^{2}}, \\
& z_{t}^{*}=\frac{1}{\varsigma(T-t)+\int_{t}^{T} \varsigma(s-t) d s} .
\end{aligned}
$$

Moreover, if $r>c(t) /(1+\theta)$, then

$$
\begin{aligned}
& \pi_{t}^{*}=M, \\
& \Pi_{t}^{*}=(1+\theta) M+1 ;
\end{aligned}
$$

if $r<c(t) /(1+\theta)$, then

$$
\begin{aligned}
& \pi_{t}^{*}=0, \\
& \Pi_{t}^{*}=1 ;
\end{aligned}
$$

if $r=c(t) /(1+\theta)$, then $\pi_{t}^{*}$ can take any value in $[0, M]$.

Remark 5. The optimal investment and debt ratio policies are verified the same as the corresponding part in [21] without considering reinsurance. So, we can see that it is the same between time-inconsistency and time-consistency for optimal investment and debt ratio policies. This is because that the investment and debt ratio policies are independent with discount rate in this model.

Remark 6. We can see that the optimal dividend payment is the same as [21] without considering reinsurance when the discount rate function $\nu(s)=\rho$.

\section{Data Availability}

There are no data used in this study.

\section{Conflicts of Interest}

The authors declare that there are no conflicts of interest regarding the publication of this article.

\section{Acknowledgments}

The research was supported by the National Natural Science Foundation of China (no. 11571198, 11501319, and 11501321), the Project of China Postdoctoral Science Foundation (no. 2015M582064), the Natural Science Foundation of Shandong (nos. ZR2015AL013 and ZR2014AM021), and the Project of Shandong Province Higher Educational Science and Technology Program (no. J15LI05). 


\section{References}

[1] S. Browne, "Optimal investment policies for a firm with a random risk process: exponential utility and minimizing the probability of ruin," Mathematics of Operations Research, vol. 20, no. 4, pp. 937-958, 1995.

[2] C. Yin and C. Wang, "The perturbed compound Poisson risk process with investment and debit interest," Methodology and Computing in Applied Probability, vol. 12, no. 3, pp. 391-413, 2010.

[3] M. Zhou, K. C. Yuen, and C.-c. Yin, "Optimal investment and premium control in a nonlinear diffusion model," Acta Mathematicae Applicatae Sinica, vol. 33, no. 4, pp. 945-958, 2017.

[4] B.-D. Finetti, "Su un 'impostazione alternativa della teoria collettiva del rischio," in Transactions of the XVth International Congress of Actuaries, vol. 2, pp. 433-443, 1957.

[5] H. U. Gerber and E. S. Shiu, "Optimal dividends: analysis with Brownian motion," North American Actuarial Journal, vol. 8, no. 1, pp. 1-20, 2004.

[6] H. U. Gerber and E. S. Shiu, "On optimal dividend strategies in the compound Poisson model," North American Actuarial Journal, vol. 10, no. 2, pp. 76-93, 2006.

[7] C. Yin and C. Wang, "Optimality of the barrier strategy in de Finetti's dividend problem for spectrally negative Lévy processes: an alternative approach," Journal of Computational and Applied Mathematics, vol. 233, no. 2, pp. 482-491, 2009.

[8] C. Yin and Y. Wen, "Optimal dividend problem with a terminal value for spectrally positive Levy processes," Insurance: Mathematics and Economics, vol. 53, no. 3, pp. 769-773, 2013.

[9] H. Meng, T. K. Siu, and H. Yang, "Optimal dividends with debts and nonlinear insurance risk processes," Insurance: Mathematics and Economics, vol. 53, no. 1, pp. 110-121, 2013.

[10] C. Yin and K. C. Yuen, "Optimal dividend problems for a jump-diffusion model with capital injections and proportional transaction costs," Journal of Industrial and Management Optimization, vol. 11, no. 4, pp. 1247-1262, 2015.

[11] B. Avanzi, "Strategies for dividend distribution: a review," North American Actuarial Journal, vol. 13, no. 2, pp. 217-251, 2009.

[12] H. Schmidli, "Optimal proportional reinsurance policies in a dynamic setting," Scandinavian Actuarial Journal, no. 1, pp. 5568, 2001.

[13] Z. Liang, K. C. Yuen, and J. Guo, "Optimal proportional reinsurance and investment in a stock market with Ornstein-Uhlenbeck process," Insurance: Mathematics and Economics, vol. 49, no. 2, pp. 207-215, 2011.

[14] H. Schmidli, "On minimizing the ruin probability by investment and reinsurance," The Annals of Applied Probability, vol. 12, no. 3, pp. 890-907, 2002.

[15] L. Bai and J. Guo, "Optimal proportional reinsurance and investment with multiple risky assets and no-shorting constraint," Insurance: Mathematics and Economics, vol. 42, no. 3, pp. 968-975, 2008.

[16] X. Zhang and T. K. Siu, "Optimal investment and reinsurance of an insurer with model uncertainty," Insurance: Mathematics and Economics, vol. 45, no. 1, pp. 81-88, 2009.

[17] J. Paulsen and H. k. Gjessing, "Optimal choice of dividend barriers for a risk process with stochastic return on investments," Insurance: Mathematics and Economics, vol. 20, no. 3, pp. 215223, 1997.

[18] H. Meng and T. K. Siu, "On optimal reinsurance, dividend and reinvestment strategies," Economic Modelling, vol. 28, no. 1-2, pp. 211-218, 2011.
[19] P. Azcue and N. Muler, "Optimal investment policy and dividend payment strategy in an insurance company," The Annals of Applied Probability, vol. 20, no. 4, pp. 1253-1302, 2010.

[20] Z. Jin, H. Yang, and G. Yin, "Optimal debt ratio and dividend payment strategies with reinsurance," Insurance: Mathematics and Economics, vol. 64, pp. 351-363, 2015.

[21] Q. Zhao, Z. Jin, and J. Wei, "Optimal investment and dividend payment strategies with debt management and reinsurance," Journal of Industrial and Management Optimization, vol. 13, no. 5, pp. 1-26, 2017.

[22] R. H. Strotz, "Myopia and inconsistency in dynamic utility maximization," Review of Economic Studies, vol. 23, no. 3, pp. 165-180, 1955.

[23] J. Marn-Solano and J. Navas, "Consumption and portfolio rules for time-inconsistent investors," European Journal of Operational Research, vol. 201, no. 3, pp. 860-872, 2010.

[24] Q. Zhao, J. Wei, and R. Wang, "On dividend strategies with non-exponential discounting," Insurance: Mathematics and Economics, vol. 58, pp. 1-13, 2014.

[25] J. Yong, "Time-inconsistent optimal control problems and the equilibrium HJB equation," Mathematical Control and Related Fields, vol. 2, no. 3, pp. 271-329, 2012. 


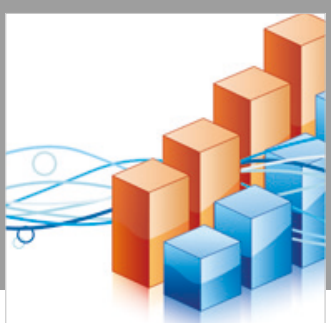

Advances in

Operations Research

\section{-n-m}
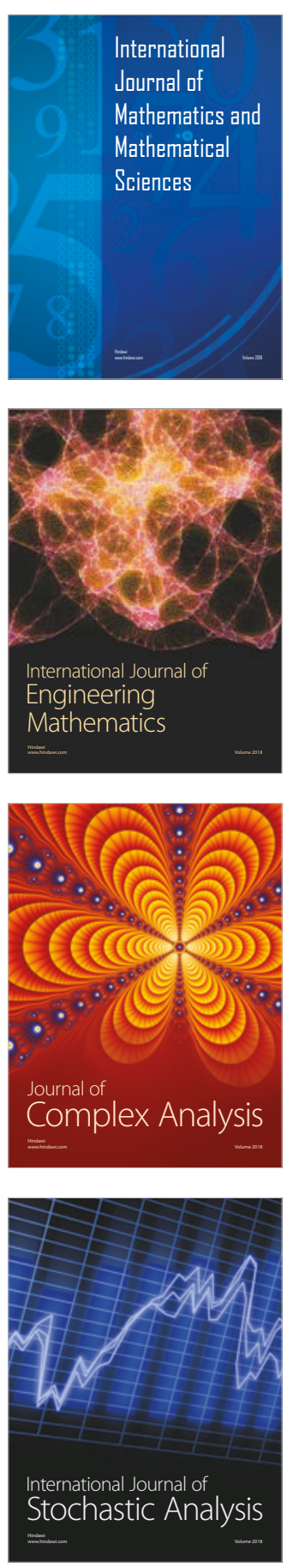
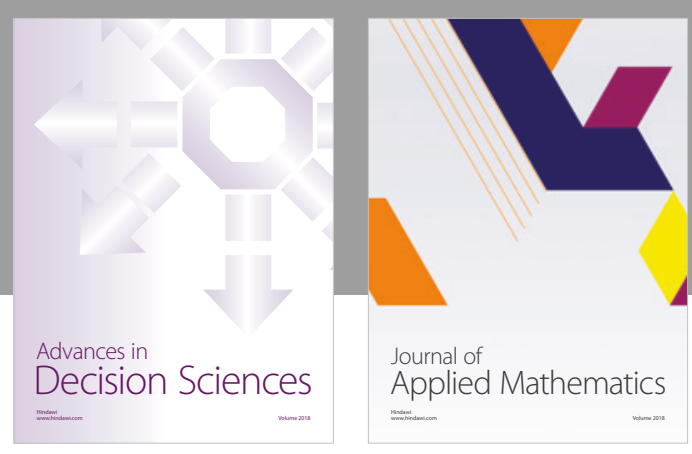

Journal of

Applied Mathematics
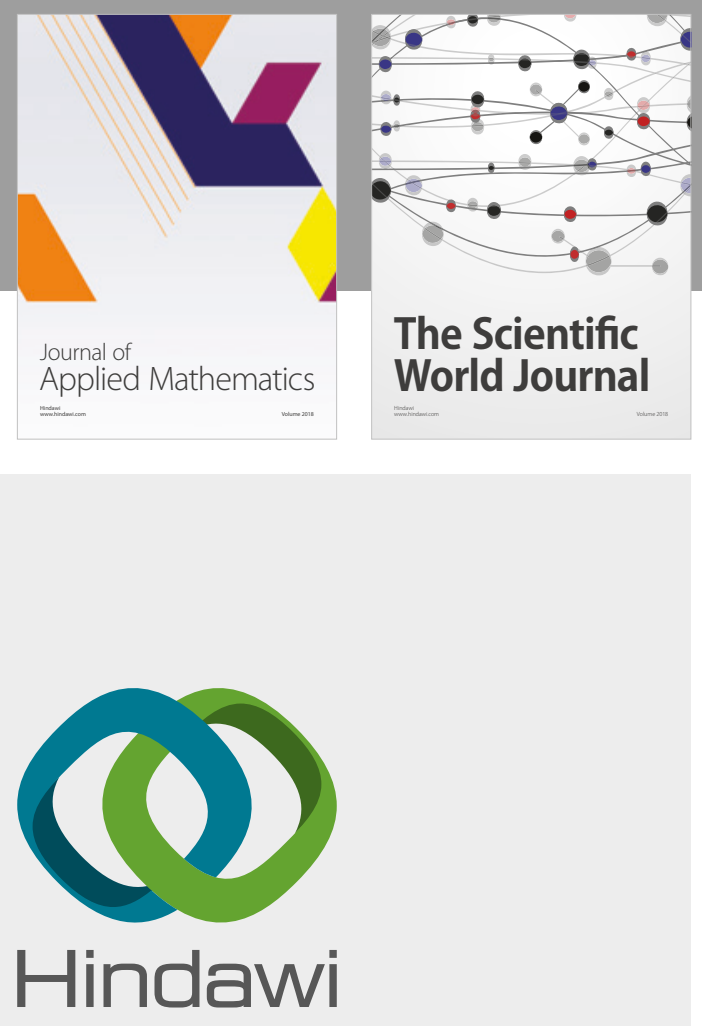

Submit your manuscripts at

www.hindawi.com

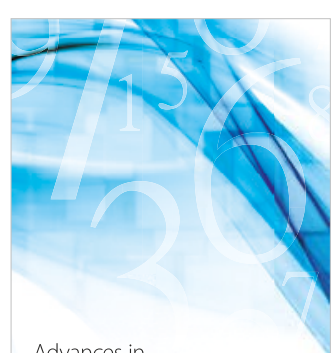

Advances in
Numerical Analysis
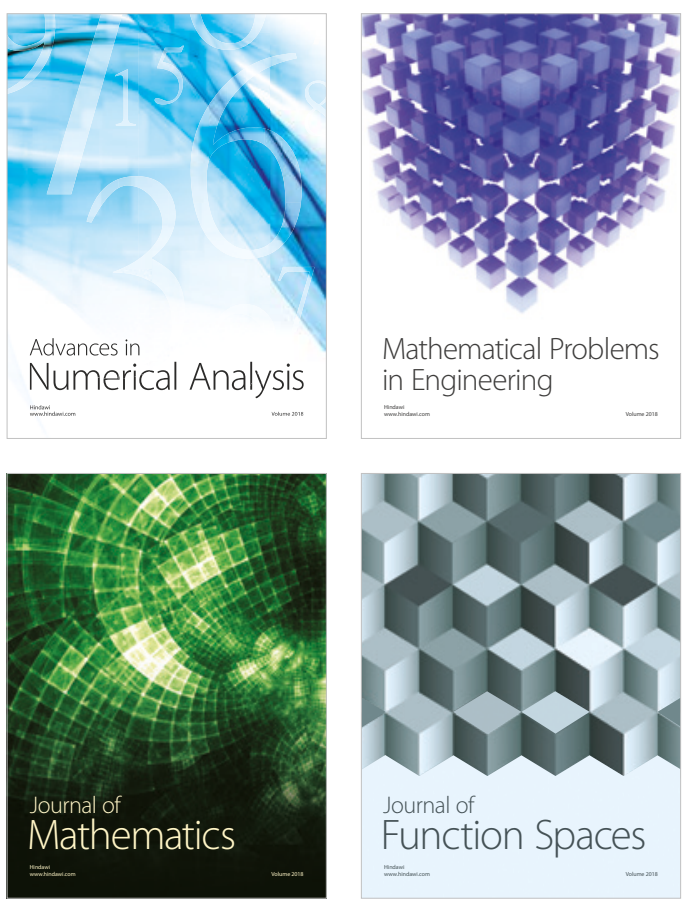

Mathematical Problems in Engineering

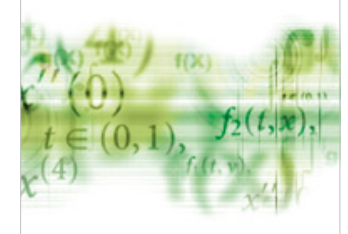

International Journal of

Differential Equations

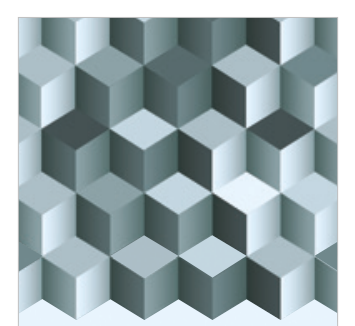

Journal of

Function Spaces

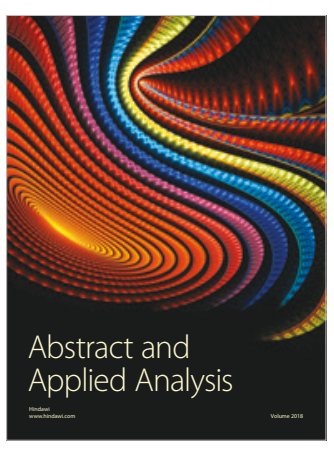

The Scientific

World Journal

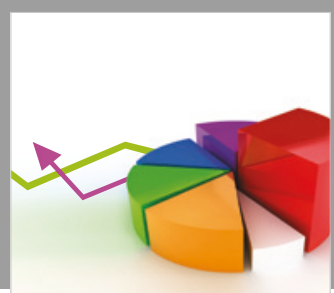

Journal of

Probability and Statistics
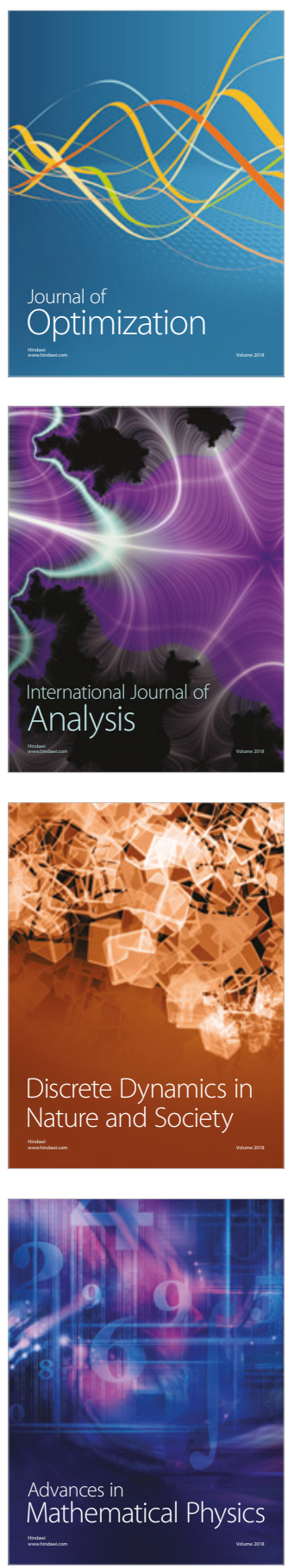